\title{
Satellite Integrated Electronic System Design from Cloud Computing View
}

\author{
ZHU Ye ${ }^{1,2}$, DAI Ya $^{1 *}$, CHENG Rui ${ }^{1}$, ZHANG Xiaojie ${ }^{1}$ \\ ${ }^{1}$ Shanghai Engineering Center for Microsatellites, Shanghai, China \\ ${ }^{2}$ Institute of geology and geophysics, CAS, Beijing, China \\ ocean_ye@126.com
}

\begin{abstract}
As system integration becomes an increasingly important challenge for satellite electronic systems, there has been a significant demand for system-level optimizing. Cloud computing is suitable for this case. Proposal a satellite integrated electronic system architecture which using the cloud computing idea and virtual machine software implementation. Base on the system requirement analyses, construct the integrated electronic system by computing unit, memory unit and interface unit. With a flexible redundant model which simplify the system design by reduce the processors number and circuit complexity, without sacrifice the reliability. Analyzed the virtual machine monitor property and satellite software requirement, and then defined the satellite software architecture base on virtual machines. After evaluations shows that from the cloud computing view design the satellite integrated electronic system can get more concise result. It decrease the hardware needed, increase the resource isolation, and breaks the one-to-one correspondence between logical systems and physical systems. Provide a new integrated electronic system design approach.
\end{abstract}

Keywords-Satellite integrated electronic system, Cloud computing, Virtual machine

\section{INTRODUCTION}

Satellite integrated electronic system (IES) is one of the five satellite platform systems, paralleling with the satellite attitude and orbit control, thermal control, energy control and motion control system. It uses the computer network technology to connect satellite onboard electronic equipment, with the purpose of achieving satellite internal information sharing and comprehensive utilization, subsystem function integration, and resources reorganization and optimization. ${ }^{[1]}$ IES aimed to break the traditional subsystem boundary and optimized the resource spending. It has been widely adopted in satellite to integrate on board data handling subsystem (OBDH), Telemetry and Telecomm and subsystem(TM/TC), Attitude and Orbit Control Subsystem (AOCS) and Power Conditioning and Distribution Unit (PDCU) together.

Cloud computing is a type of Internet-based computing that provides shared computer processing resources and data to computers and other devices on demand. It is a model for enabling ubiquitous, on demand access to a shared pool of configurable computing resources. ${ }^{[2]}$ Cloud computing technology has been applied to many commercial systems, this paper introduce it to spacecraft electronic system designs. Considering all on-board computing resources optimizing aims to improve reliability, reduce power consumption and device weight. Drawing on the idea of virtualization in cloud computing and the concept of flexible design, propose the satellite IES based on cloud computing.

\section{SATELLITE INTEGRATED ELECTRONIC SYSTEM REQUIREMENT ANALYSES}

Cloud computing considering all function as a service, software as a service (SaaS), infrastructure as a service (IaaS) and platform as a service (PaaS). Illustarted by the example of the Dark matter particle detector satellite (Dampe), analysis of computing and storage requirements from a service perspective.

\section{A. Traditional electronic system requirement analyses}

Dampe was launched at 2015/12/17, it integrated electronic system design diagram is shown in Fig.1. Although it integrates the attitude control computer and the OBDH computer together, it still doesn't break the boundary of the subsystems and instruments.

The function satellite needed include: high reliability task management, TM/TC process, AOCS, PDCU, payload data handling. Correspond to Dampe satellite by configure OBDH computer, power manage unit, attitude computer(integrated into OBDH), TMTC router(integrated into OBDH), Star track data handling unit, fiber optic gyroscope data handling unit to fulfill the service needed. Dampe needs to work for a long life, the platform adopt a full redundancy scheme, using 8 processor modules and 2 FPGA modules. And it computing, memory and interface needed illustrated in table I.

TABLE I. DAMPE SATELLITE PLATFORM ELECTRICAL COMPUTING MEMORY AND INTERFACE REQUIREMENT

\begin{tabular}{|c|c|c|c|}
\hline Device & Num. & Computing & Memory \\
\hline $\begin{array}{l}\text { Star } \\
\text { Trackers }\end{array}$ & 3 & $\begin{array}{l}\text { Image processing, } \\
\text { attitude } \\
\text { determination } \\
\text { 100MIP's }{ }^{[3]}\end{array}$ & $\begin{array}{l}\text { Program: } 6 * 512 \mathrm{~K} * 8 \mathrm{bit} ; \\
\text { Data Memory: } 2 * 128 \mathrm{~K} * 8 \mathrm{bit} \\
\text { EEPROM: } 2 * 128 \mathrm{~K} * 8 \mathrm{bit} \\
\text { CCD Image: } 512 \mathrm{~K} * 16 \mathrm{bit}\end{array}$ \\
\hline FOG & 2 & $\begin{array}{l}\text { ADC, closed loop } \\
\text { algorithm and DACs } \\
\text { DSP(TIVC33,60MI } \\
\text { Ps/120MFLOPs) }\end{array}$ & Memory:8Kbytes \\
\hline SMU & 2 & $\begin{array}{l}\text { CPU:ERC32(20 } \\
\text { MIPs/5 MFLOPs }\end{array}$ & $\begin{array}{l}\text { Program:4*256K*8bit; } \\
\text { Data Memory:4*256K*8bit } \\
\text { PROM:128K Bytes }\end{array}$ \\
\hline PCDU & 2 & CPU:80C32 & Memory:8Kbytes \\
\hline GPS & 1 & $\begin{array}{l}\text { TMS320VC33 } \\
\text { APA600 }\end{array}$ & \\
\hline ADU & 2 & APA600: $\quad$ System & RAM:126K \\
\hline PIU & 2 & Gates:600000 & Registers: 21504 \\
\hline TCU & 2 & & \\
\hline
\end{tabular}




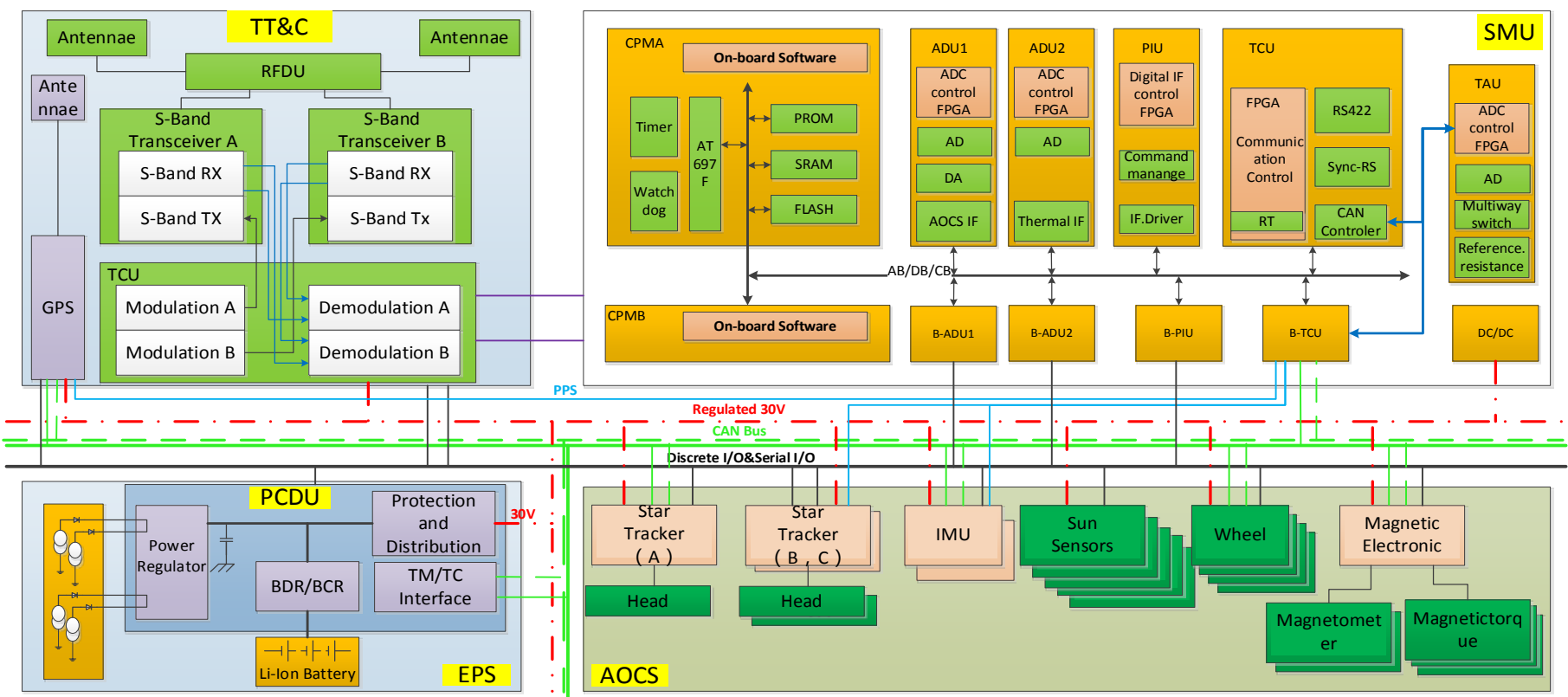

Fig. 1. Dampe platform electronic architecture

\section{B. Cloud computing perspective requirement}

In accordance with the ideas of cloud computing to redefine the needs of satellite integrated electronics. Roughly divided into computing, memory and Communication/Interface.

In traditionally, the system is divided into different part first, and then the computation, storage and interface requirements are completed in the single machine level. At the cloud computing point always analysis from the system view, and considering not only one machine needed but also satellite needed at different work mode. Then review the Dampe processers and FPGA needed. According to the working mode of the satellite, the requirement of the activity device is shown in table II.

TABLE II. PROCESSER REQUIREMENT OF THE DIFFERENT WORKING MODE

\begin{tabular}{|c|c|c|c|c|}
\hline & \multicolumn{2}{|c|}{ High-precision attitude mode } & \multirow{2}{*}{$\begin{array}{c}\text { Payload } \\
\text { standby } \\
\text { mode }\end{array}$} & \multirow{2}{*}{$\begin{array}{l}\text { Safe } \\
\text { mode }\end{array}$} \\
\hline & $\begin{array}{c}\text { Double Star } \\
\text { Tracker }\end{array}$ & $\begin{array}{c}\text { Star tracker } \\
\text { and FOG }\end{array}$ & & \\
\hline $\begin{array}{l}\text { Star } \\
\text { Trackers }\end{array}$ & 2 & 1 & 0 & 0 \\
\hline FOG & 0 & 1 & 0 & 0 \\
\hline SMU & 1 & 1 & 1 & 1 \\
\hline PCDU & 1 & 1 & 1 & 1 \\
\hline GPS & 1 & 1 & 0 & 0 \\
\hline ADU & 1 & 1 & 1 & 1 \\
\hline PIU & 1 & 1 & 1 & 1 \\
\hline TCU & 1 & 1 & 1 & 1 \\
\hline
\end{tabular}

Based on this, the computing and storage capacity of satellite equipped is much greater than the actual demand. Cause every device considering redundant alone, then the system have much redundant, and those backup device cannot share their computing and storage ability. From a system views the attitude calculation is much like the calculation used while the star track finding stars, but in the traditional way they using different device or using a processor which is not adapted at those calculation.

While the satellite running in different modes, its need different resource. From cloud computing view the resource should allocated on-demand. Here we define satellite resource requirement at variable level: Full-mode means the satellite working at maximum load, all the computing and storage resources are fully utilized under the condition of system level margin. Ordinary-mode means the satellite's most common device working properly and satellite doesn't need adjust rapidly. In this mode, the main computing resources of the satellite cooperate to play a role, and the key resources are fully backed up. Standby-mode means the satellite only need platform working and waiting for the ground command to set up a new task. In this mode, some devices can be turned off to save powers, whilst satellite also have a fully redundancy computing resource.

After these analysis taken the Dampe as an example, we need 2 CPU and DSP as the main computing ability provider, only about half of the process it is originally needed.

\section{CLOUD COMPUTING PERSPECTIVE ELECTRONIC SYSTEM DESIGN}

The National Institute of Standards and Technology's definition of cloud computing identifies "five essential characteristics" $^{[5]}$. Three of them are very suitable for Satellite integrated electronic system's design:

- On-demand self-service. A consumer can unilaterally provision computing capabilities, such as server time and network storage, as needed automatically without requiring human interaction with each service provider.

- $\quad$ Resource pooling. The provider's computing resources are pooled to serve multiple consumers using a multitenant model, with different physical and virtual 
resources dynamically assigned and reassigned according to consumer demand.

- Rapid elasticity. Capabilities can be elastically provisioned and released, in some cases automatically, to scale rapidly outward and inward commensurate with demand. To the consumer, the capabilities available for provisioning often appear unlimited and can be appropriated in any quantity at any time.

Design of integrated electronic system based on the above characteristics.

\section{A. Integrated electronic system architecture}

The central idea of cloud computing is to calculate and optimize resource allocation, for the satellite, the electronic products can be classified as two types, sensors and processors. Define all the data storage and processing as cloud resources within the satellite. And define all sensors as the client, including the ground terminals. Taking into account the satellite operating mode and computing needs, the hardware architecture as shown in Fig.2.

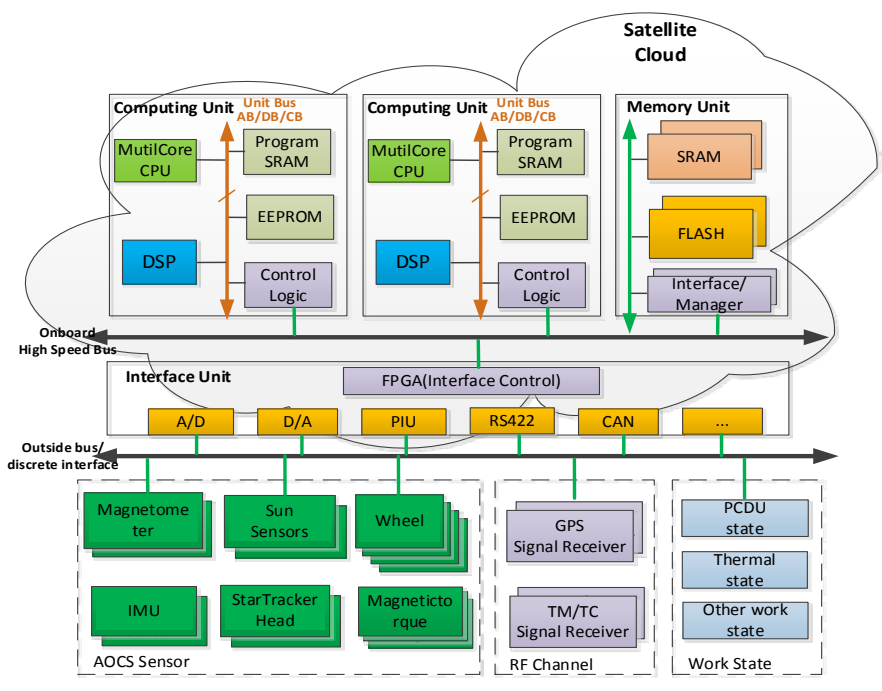

Fig. 2. Cloud computing satellite electronic architecture

Configurations of two sets of computing units, which include a CPU as main processor and a DSP as coprocessor, backup and work together. Under the full-mode working mode, two sets of DSP are needed to complete the data processing of IMU, GPS and star trackers, and integrated some attitude calculation missions. Configure a piece of CPU to complete the tasks such as satellite state estimation, telemetry data management and work mode setting. The operation between the two computing units can dynamically migrate through the suitable software implement. In this way, the system backup scheme is realized. The memory unit is used for all telemetry data and application data. It also supports large capacity data buffering, such as image data from the star trackers. The memory unit comprises two backup management and interface control modules, which can fulfill the high reliability of the system through the optimized configuration of the capacity and the memory chips. Interface unit is a channel for cloud computing and storage capabilities to exchange data with satellite sensors which include the AOCS sensor, RF channel and satellite state acquire sensor.

The information transmission in the integrated electronic system of the cloud architecture includes:

- External bus which transfers data between the satellite sensors and interface unit. It has no uniform definition, can be CAN, RS422 or discrete interface. Through the reasonable configuration of the interface unit, we can realize the unified interface for computing unit or memory unit. The FPGA inside the interface unit should map all external information into the corresponding inside resource.

- Architecture bus which transfers data between the computing unit, memory unit and interface unit. The high speed data transmission can be realized by using the universal high speed bus (e.g. Space Wire, TLK2711), which is the base of dynamic computing migration.

- Unit bus which transfers control, data and address information inside the unit. It using the traditional data control and address bus as the basic architecture.

The integrated electronic architecture based on cloud computing realizes the separation of sensing and processing, so that designers are more focused in the field of professional work. On the basis of centralized computing, the parallel processing based on multi-core processor and distributed computing based on multi-processor are designed. On the basis of the unified application data view, it can reduce the influence of time delay, data synchronization and other factors. Unified computing and storage requirements planning, breaking the boundaries of single machine, data processing in a common view, reducing the pressure of data transfer between devices. Comprehensive redundancy backup makes the whole system more reliable.

\section{B. Integrated software virtualization}

There is a need for parallel processing in satellite software, for examples, high precision attitude calculation which requires the sensor data acquire as quickly as it can and as short delay as it can. In the payload data processing, it is also concerned with the synchronization of the clock signal and the track, attitude, temperature data and payload sensor data. Virtualization technology is a kind of technology for the abstract simulation of computer resources, with the advantages of isolation, consolidation and migration ${ }^{[6]}$. The system level virtualization technology introduces a new abstraction layer between the hardware and the operating system to become virtual machine monitor (VMM). The hardware resources are taken over by VMM, and the application program of the original single core processor is transplanted to multi-core platform, so as to provide support for parallel processing. Therefore, on the basis of the design of the integrated electronic hardware system based on multi-core processor and DSP as the coprocessor, virtualization technology is introduced as the basis of the software design of the integrated electronic system.

System level virtualization technology can be divided into full virtualization and paravirtualization according to the 
degree of virtualization. Full virtualization requires a powerful processor, and when the virtual machine running, its need more resource. The biggest feature of paravirtualization and full virtualization is that it does not need to capture privileged commands, so it has higher efficiency. But guest programs need to be specifically modified to run in this environment. While in the embedded situation, RTOS usually need to be changed, so the paravirtualization is more applicable.

RT-Xen demonstrates the feasibility, efficiency, and efficacy of fixed-priority hierarchical real-time scheduling in virtualized platforms ${ }^{[7]}$. When considering the design of integrated electronic software architecture, take full account of it property that Virtualization breaks the one-to-one correspondence between logical systems and physical systems, allows subsystem development to be done separately. Cloud architecture integrated electronic software architecture as shown in fig.3.

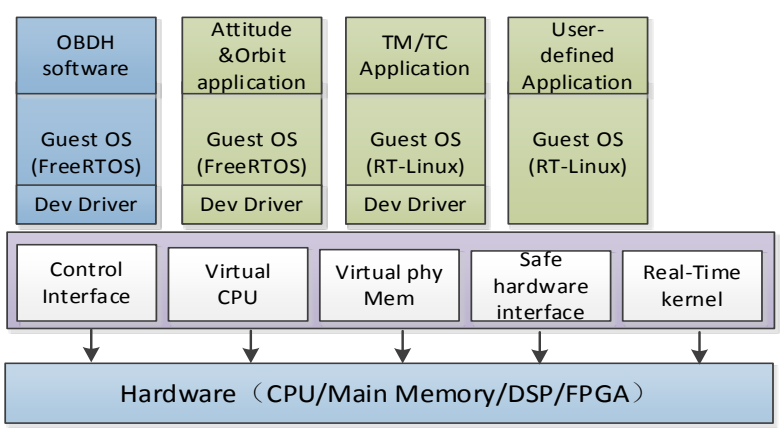

Fig. 3. Software architecture base on virtual machines

Xen is an open source paravirtualization solution, its interface fall into three broad aspects of the system: memory management, the processor and hardware interface. To support real-time systems, a virtual machine monitor (VMM) integrated a real-time kernel to ensure the integrated subsystems meet the response requirements. Through the virtualization way give every guest OS a virtual CPU and constant memory, while the VMM manage the virtual memory table. Xen exposes a set of device abstractions, which allows us to design an interface driver, fulfill the protection and isolation require. I/O data is transferred between each domain using shared-memory via VMM.

Define the OBDH as the domin_0 system it will be created at boot time. As the main application is permitted to use the control interface which provides the ability to create and terminate other domains just like the common OBDH application do. Not only manage the processor and memory resources, the control interface also manage the $\mathrm{I} / \mathrm{O}$ devices. Integrate all attitude and orbit manage application which include the attitude sensor data process, orbit prediction, attitude control manage, etc., together running in a guest OS. OBDH doesn't control the attitude sensor directly anymore but through the attitude manage application running on it-self's guest OS. TM/TC manage application using a C-S(ClientService) mode organize all telemetry data and telecommand data. It running in a guest $\mathrm{OS}$ as the server and other application no matter running on which guest OS can send data or get data from the TM/TC application through the VMM layer. This way all application's state can organized in a separated way and support it can be developed at same time. While some other requirement, like payload management, thermal control, etc., can be implemented by creating a customized guest OS and running user defined application.

All application deployment on the virtual machine can be transplanted to the other virtual machine without any changes. The integrated electrical system has two parallel computing units; it can be working in a cold redundant way or can be working at same time running different application. For example the computing unit A can running the OBDH-A and attitude \& orbit manage application, while the computing unit B running the OBDH-B and TM/TC manage application. When some error detected the application can be transplanted directly to the redundant one.

\section{CONCLUSION}

Data process on-board is the tendency of the satellite utility pattern, and from the cloud computing point everything is a service. We define a satellite as a platform and will provide service for the ground user. Inside it will be divided as sensor and processor, get environment state directly by sensor and manipulate all data to knowledge by processor. By recognizes computing requirement, design a compact architecture which breaking the subsystem constraint allows resource provisioning and utilizing separately. Compare to traditional design it resources can be shrunk to have only two redundant computing unit, one centralized memory unit and redundant interface unit. The result is an appropriately provisioned system without unnecessary cost or complexity. The introduction of the concept of virtualization makes it possible to abstract the hardware as a uniform perspective, so that system designers and software designers are all see the same storage and computing resource. This way the algorithm has the advantages of generality and high reuse rate, and does not need to consider which specific device it implemented on.

\section{REFERENCES}

[1] J.C. Chiarini. M.Perren. J.M.Nakache. S.Palumbo. The Galileo Satellite [A]. 21st International Communications Satellite system Conference and Exhibit. AIAA, 2003-2306[C].

Peter Mell and Timothy Grance (September 2011). The NIST Definition of Cloud Computing. (Technical report). National Institute of Standards and Technology: U.S. Department of Commerce. doi:10.6028/NIST.SP.800-145. Special publication 800-145.

[2] Schmidt U.: Intelligent error correction method applied on active pixel sensor based star tracker. Proceedings of SPIE Vol. 5964, (2005)

[3] M. Inc. Atmel (4148-AERO-12/03), TSC695F SPARC 32-bit Space Processor User Manual. 2003

[4] Peter Mell and Timothy Grance (September 2011). The NIST Definition of Cloud Computing (Technical report). National Institute of Standards and Technology: U.S. Department of Commerce. doi:10.6028/NIST.SP.800-145. Special publication 800-145.

[5] Rich Ublig, Gil Neiger, et al. Intel Virtualization Technology. IEEE Computer Magazine, 2005, 38(5):48-56.

[6] S.H. VanderLeest. ARINC 653 Hypervisor. Proceedings of 29th Digital Avionics Systems Conference of IE-EE/AIAA. Salt Lake City, 3-7 October 2010, pp. 1-20.

[7] Sisu Xi, Justin Wilson, Chenyang Lu, and Christopher Gill. RT-Xen: Towards Real-time Hypervisor Scheduling in Xen [J]. Embedded Software, 2011: 39-48. 